\title{
Aberrant expression of RSK4 in breast cancer and its role in the regulation of tumorigenicity
}

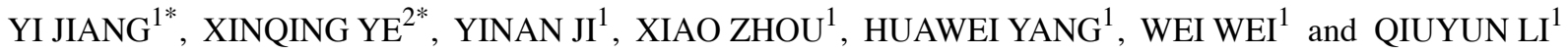 \\ Departments of ${ }^{1}$ Breast Surgery and ${ }^{2}$ Pathology, The Affiliated Tumor Hospital of \\ Guangxi Medical University, Guangxi, Nanning 530021, P.R. China
}

Received February 8, 2017; Accepted June 30, 2017

DOI: 10.3892/ijmm.2017.3069

\begin{abstract}
Breast cancer is the most frequently diagnosed cancer in both more and less economically developed countries and remains the leading cause of cancer-related death in women worldwide. In this study, to explore the expression and pathological role of RSK4 in breast cancer progression, we demonstrated that RSK4 expression was significantly decreased in breast cancer cells and tissues, and the overexpression of RSK4 in MDA-MB-231 cells inhibited cell migration and invasion. In a mouse model experiment, overexpression of RSK4 in mice further confirmed its critical role in regulating breast cancer tumorigenicity. The regulatory role of RSK4 in breast cancer development was mediated by AKT and extracellular signal-regulated kinase (ERK) signaling pathways and the expression of RSK4 was altered by DNA methylation in promoter regions. These results provide important insight into the role of RSK4 in cancerogenesis and may help to improve the prevention, diagnosis and treatment of breast cancer.
\end{abstract}

\section{Introduction}

Breast cancer is the most frequently diagnosed cancer in both more and less economically developed countries and remains the leading cause of cancer-related death in women worldwide (1). Although the breast cancer incidence and death rate have been greatly reduced in developed countries such as the US and the UK due to early detection and improved treatment, its incidence and mortality rate in South America, Africa and Asia have been rising possibly due to the reproductive pattern change, increased obesity or lack of physical activity and delayed application of early screening (1). According to the

Correspondence to: Dr Qiuyun Li, Department of Breast Surgery, The Affiliated Tumor Hospital of Guangxi Medical University, 71 Hedi Road, Guangxi, Nanning 530021, P.R. China

E-mail: 1i_qiuyun2016@sina.com

*Contributed equally

Key words: RSK4, breast cancer, AKT, extracellular signal-regulated kinase, DNA methylation, tumorigenicity latest data released by the National Cancer Center of China, the crude mortality rate of female breast cancer in registration areas was $10.24 / 100,000$ in 2009 , accounting for $54 \%$ of all female cancer mortality (2). To provide early detection and more effective therapy for breast cancer, comprehensive understanding of the molecular mechanisms mediating breast cell transformation is crucial.

It has been shown that X-linked genes are involved in the development of breast cancer (3). Among these, the X-linked ribosomal S6 kinase RPS6KA6 (RSK4) was first identified as a commonly deleted gene in complex X-linked mental retardation patients (4) and its ubiquitous expression in a number of cell types suggested its involvement in multiple biological and pathological processes such as murine embryogenesis, fibroblast growth and diabetes development (5-8). RSK4 expression was found to be downregulated in several types of tumors such as colon carcinomas, renal cell carcinomas, colorectal adenomas, endometrial cancer and ovarian cancer (9-11). Furthermore, overexpression of RSK4 was found to induce cell cycle arrest and senescence features while RSK4 inhibition resulted in bypass of stress or oncogene-induced senescence, suggesting its function as a potential tumor-suppressor gene $(9,12)$. More importantly, the expression of RSK4 was also found to be aberrantly regulated in breast cancer cells (3). In a recent study of breast cancer patient specimens, RSK4 expression was found to be significantly decreased in breast cancer tissues in comparison with non-cancerous tissues (13). Further investigation also showed that the downregulation of RSK4 expression in breast cancer cells is mediated by gene expression inhibition through promoter hypermethylation, which frequently occurs in breast cancer cells (13). Consistently, RSK4 knockdown in human breast adenocarcinoma cells significantly promoted proliferation and migration, which further confirms the role of RSK4 in the development and progression of breast cancer (14).

In human breast adenocarcinoma cells with silenced RSK4 gene expression, the mRNA expression levels of RSK4 and E-cadherin were significantly downregulated while the expression of Ki-67, cyclin D1 and CXCR4 was markedly increased. These results suggest that these genes are in the downstream signaling during RSK4-regulated cell fate decision (14). The 90-kDa ribosomal S6 kinases (RSKs) belong to serine kinases that are activated by growth factors through the Ras-dependent mitogen-activated protein (MAP) kinase 
cascade including MEK and extracellular signal-regulated kinase (ERK) (7). Previous studies have also shown that RSK4 is one of the ERK substrates and functions as a target of the ERK signaling pathway $(7,15)$. Moreover, it has also been demonstrated that RSK4 inhibits the transcriptional activation of specific targets of RTK signaling, as well as the activation of ERK in mice, and RSK4 expression inversely correlates with the presence of activated ERK 1/2 (6). Although the expression and function of RSK4 in tumorigenesis have been confirmed by several studies, its expression during breast cancer development and potential application as a breast cancer biomarker need further validation.

In this study, we showed that RSK4 expression was significantly suppressed in cell lines (MCF-7, T47D, ZR-75-1, MDA-MB-436, SK-BR-3 and MDA-MB-231) and breast cancer tissues. In addition, RSK4 overexpression in MDA-MB-231 cells effectively inhibited proliferation, migration and invasion. Moreover, overexpression of RSK4 in a breast cancer mouse model significantly suppressed breast cancer progression. Furthermore, we also demonstrated that the AKT and ERK signaling pathways and epithelial mesenchymal transition (EMT) were involved in the RSK4-regulated breast cancer development, and the reduced expression of RSK4 in MDA-MB-231 cells may be attributed to DNA methylation in its promoter region. These results indicate that RSK4 may function as a valuable biomarker for the study of breast cancer progression and may have translational application for the early detection and novel therapy of breast cancer.

\section{Materials and methods}

Ethics statement. The present study was approved by the Ethics Committee of Guangxi Medical University, and we received informed consent forms from all patients.

Cell lines and tissues. MCF-10A (non-malignant) and MCF-7, T47D, ZR-75-1, MDA-MB-436, SK-BR-3 and MDA-MB-231 human breast cancer cell lines were kindly provided by the Affiliated Tumor Hospital of Guangxi Medical University. The non-cancerous matched tissue and cancerous tissue samples were obtained from 8 patients with breast cancer after breast-reductive surgery in the Affiliated Tumor Hospital of Guangxi Medical University, and detailed information (including age, diagnosis, TNM, stage and histological grade) on the 8 patients is shown in Table I.

Gel-based RT-PCR and real-time RT-PCR. Firstly, we detected the mRNA expression level in cell lines and four tissue samples (normal and cancerous) from 8 patients with breast cancer. Total RNA of the cells and tissue samples was extracted using TRIzol reagent according to the manufacturer's instructions (Thermo Fisher Scientific, Carlsbad, CA, USA). Firstly, total RNA clean up was performed with the RNeasy Mini kit (Qiagen, Inc., Valencia, CA, USA), then $210 \mu \mathrm{l}$ of $100 \% \mathrm{EtOH}$ and $50 \mu \mathrm{l}$ of RNase/DNase-free water were added to $40 \mu \mathrm{l}$ of the RNA sample followed by the addition of buffer RLT containing $1 \% \beta$-mercaptoethanol. Prior to RT-PCR, per sample, $5 \mu \mathrm{l}$ of the RNA sample prepared above was diluted 100 -fold using RNase-free TE buffer ( $5 \mu 1$ of RNA $+95 \mu 1$ of RNase-free TE buffer; Takara Bio, Inc., Otsu, Japan) in a 96-well plate. The reaction system of RT-PCR was conducted in a final volume of $20 \mu \mathrm{l}$ containing $8 \mathrm{ng}$ of RNA ( $2 \mathrm{ng} / \mu \mathrm{l})$ and $12 \mu \mathrm{l}$ of $1 \mathrm{X}$ concentration of Bio-Rad iScript Onestep RT-PCR mix with SYBR-Green (Bio-Rad, Berkeley, CA, USA) and $4 \mu \mathrm{l}$ of primer mix. The RT-PCR reaction condition was $94^{\circ} \mathrm{C}$ for $2 \mathrm{~min}$, followed by 30 cycles of $94^{\circ} \mathrm{C}$ for $30 \mathrm{sec}, 55^{\circ} \mathrm{C}$ for $1 \mathrm{~min}$ and $68^{\circ} \mathrm{C}$ for $2 \mathrm{~min}$ and a final extension cycle was carried out at $68^{\circ} \mathrm{C}$ for $5 \mathrm{~min}$. The GAPDH gene was used as an internal control. The RT-PCR product was separated on $2 \%$ agarose gels and the gel image was analyzed and quantified using ImageQuant software version 5.2 (Molecular Dynamics, Sunnyvale, CA, USA). Secondly, after measurement of the RNA concentration and reverse transcription, mRNA expression levels were determined by real-time RT-PCR using an ABI PRISM 7900HT sequence detection system (Applied Biosystems Life Technologies, Foster City, CA, USA). Three independent biological repeats were performed and the GAPDH gene was used as an internal control. The primers used for RSK4 gene and GAPDH were RSK4 F, 5'-TGAGTGGTGGAAACTGGGACA ATA-3' and R, 5'-TGGCATGGACTGTGGTCATGAGTC-3'; GAPDH F, 5'-GCACCGTCAAGGCTGAGAAC-3' and R, 5'-TGGTGAAGACGCCAGTGGA-3'. First-strand cDNA was reversely transcribed using Primerscript II 1st Strand cDNA Synthesis kit (no. 6210A; Takara Bio, Inc.). The Takara kit (no. RR390A; Takara Bio, Inc.) was used for real-time RT PCR, which was conducted in a $20 \mu 1$ reaction system with $10 \mu 12 \mathrm{X}$ mix buffer, forward and reverse primers $(0.4 \mu 1$ each), $1 \mu \mathrm{l}$ cDNA, and $15.4 \mu 1$ double distilled water. The real-time RT-PCR was performed using the following conditions: $94^{\circ} \mathrm{C}$ for $5 \mathrm{~min}$, 30 cycles of denaturation at $94^{\circ} \mathrm{C}$ for $1 \mathrm{~min}$, annealing at $55^{\circ} \mathrm{C}$ for $1 \mathrm{~min}$, and extension at $72^{\circ} \mathrm{C}$ for $40 \mathrm{sec}$. The $2^{-\Delta \Delta \mathrm{Cq}}$ method was applied for quantification of the relative expression of target genes.

Western blot analysis. For protein extraction, cultured breast cancer cell lines were first lysed in cell lysis buffer (140 mM $\mathrm{NaCl}, 10 \mathrm{mM}$ Tris-HCl, $1 \%$ Triton X-100, 1 mM EDTA, $1 \mathrm{X}$ protease inhibitor cocktail). For protein extraction in breast tissues, collected breast tissues were first homogenized in liquid nitrogen, and then treated with the same cell lysis buffer. After determination of protein concentrations by the BCA method, protein samples were separated by SDS-PAGE and transferred to polyvinylidene difluoride (PVDF) membranes (Millipore Corp., Billerica, MA, USA), followed by blocking with $5 \%$ bovine serum albumin (BSA) for $2 \mathrm{~h}$, and incubation with the primary antibody for $1 \mathrm{~h}$ at room temperature, including anti-phosphorylated (p)-AKT (cst4051, 1:200), anti-AKT (cst9272, 1:1,800), p-ERK (cst9101, 1:1,000), ERK (cst9102, 1:1,000), anti-E-cadherin (cst9961, 1:1,000) and anti-vimentin (cst3932, 1:500) (Cell Signaling Technology, Inc., Danvers, MA, USA). After being washed with TBST for 3 times, the membranes were then incubated with peroxidase-conjugated secondary antibodies for $1 \mathrm{~h}$. Band intensity was determined used chemiluminescent reagents (Pierce; Thermo Fisher Scientific, Waltham, MA, USA) and analyzed by ImageJ software v1.48 (National Institutes of Health, Bethesda, MD, USA).

Construction of stably overexpressing RSK4 in MDA-MB-23I cells. We constructed the lentiviral expression vector of the 
Table I. Detailed information of the eight breast cancer patients.

\begin{tabular}{|c|c|c|c|c|c|}
\hline No. & Age (years) & Diagnosis & TNM & Stage & Histological grade \\
\hline 1 & 52 & Invasive ductal carcinoma & T1N1M0 & I & I \\
\hline 2 & 54 & Invasive ductal carcinoma & T1N3M0 & $\mathrm{I}$ & I \\
\hline 3 & 72 & Invasive ductal carcinoma & $\mathrm{T} 2 \mathrm{~N} 1 \mathrm{M} 1$ & II & II \\
\hline 4 & 63 & Carcinosarcoma & $\mathrm{T} 2 \mathrm{~N} 2 \mathrm{M} 1$ & IV & III \\
\hline 5 & 73 & Invasive ductal carcinoma & T1N0M0 & I & I \\
\hline 6 & 31 & Invasive ductal carcinoma & $\mathrm{T} 2 \mathrm{~N} 1 \mathrm{M} 0$ & II & I \\
\hline 7 & 58 & Invasive ductal carcinoma & $\mathrm{T} 2 \mathrm{~N} 1 \mathrm{M} 1$ & III & II \\
\hline 8 & 60 & Invasive ductal carcinoma & T1N1M0 & $\mathrm{I}$ & I \\
\hline
\end{tabular}

RSK4 gene and the MDA-MB-231 cell line which stably overexpressed the RSK4 gene, following the procedure as previously described by Roy and Jacobson (16). The RSK4 gene was synthesized by the gene synthesis method and amplified by PCR, and then cloned into the pLVEF-1a/GFP-Puro vector to construct the pLVEF-1a/ GFP-Puro-RSK4 lentiviral vector. After restriction enzyme analysis and sequence identification, the lentiviral vector was packaged and the titer was detected. The human breast cancer MDA-MB-231 cells for transfection were cultured in DMEM media containing $10 \%$ FBS, sodium pyruvate and antibiotics. These cells were transfected with the recombinant lentiviral vector and cultured selectively by puromycin to acquire stably transfected cells. MDA-MB-231 cells which expressed GFP were observed by fluorescence microcopy. After $48 \mathrm{~h}$ of transfection, the expression levels of RSK4 mRNA and protein in the transfected MDA-MB-231 cells were detected by real-time quantitative PCR and western blot analysis, respectively. MDA-MB-231 cells (the untreated group, control), MDA-MB-231 cells transfected with blank vectors (the vector group) and MDA-MB-231 cells overexpressing RSK4 proteins (the MDA-MB-231-RSK4 group) were used for further analysis, and MDA-MB-231 cells transfected with blank pEGFP vectors were applied as control.

Colony formation assay. For analysis of cell proliferation potential, the untreated group, vector group and MDA-MB-231-RSK4 cells were separately seeded at 500 cells $/ 6-\mathrm{cm}$ dish in complete medium. After growing in the dish for 3 weeks, three groups of cells were fixed and visualized by staining with $0.1 \%$ crystal violet $(\mathrm{w} / \mathrm{v})$ in $20 \mathrm{nM}$ 4-morpholinepropanesulfonic acid. Visible colonies of each group were compared by counting the number of cells in each colony and three biological replicates were performed.

Cell migration and invasion assays. Breast cancer cell migration and invasion assays were performed as previously described (17). Briefly, the cell migration assay was carried out in Boyden chambers without coating of Matrigel. Migrated breast cancer cells were stained and counted. Cell invasion analysis was performed in a Boyden chamber with polyethylene terephthalate filter inserts. Three groups of breast cancer cells were plated in BSA-DMEM and inserted into the upper chamber, and the lower chamber was filled with FCS-DMEM. The invaded cells were then treated with fixation and staining solution containing
$0.1 \%$ crystal violet, $1 \%$ formalin, and $20 \%$ ethanol. Invaded breast cancer cells were stained and counted.

DNA methylation sequencing. For prediction of DNA methylation in the RSK4 gene, the DNA methylation in the RSK4 gene was analyzed by using bisulfite direct sequencing assay, and for primers specific to bisulfite-treated DNA, the DNA region of the RSK4 gene was analyzed by using the free web program MethPrimer (http://www.urogene.org/cgi-bin/ methprimer/methprimer.cgi). MethPrimer determines the presence and location of CpG islands within the RSK4 DNA sequence. Direct bisulfite sequencing for analysis of DNA methylation levels is a technique that was developed in order to determine $\mathrm{CpG}$ site-specific changes. Determination of DNA methylation changes is fundamental to understanding control of RSK4 expression within breast cancer cells and how these changes affect tumorigenesis. Prior to bisulfite conversion and sequencing, the total DNA samples were extracted from MDA-MB-231 cells and the quality of extracted DNA was assessed using the Qubit Platform (Invitrogen Life Technologies, Carlsbad, CA, USA). By treating DNA samples with bisulfite, the cytosine residues were converted to uracil while 5-methylcytosine residues remained unaffected, thus methylated and unmethylated residues in the RSK4 promoter regions were recognized by sequencing. Following bisulfite treatment, PCR amplification was performed with bisulfite-compatible primers which target a specific genomic region. During the PCR amplification, cytosine bases which are converted to uracil during bisulfite treatment are converted to thymine. Both forward and reverse primers were used at the concentration of $10 \mu \mathrm{M}$. A mix of $10 \mu \mathrm{l}$ of SYBR-Green Supermix (Bio-Rad) was prepared with $8 \mu \mathrm{l}$ of nuclease free water, plus $0.5 \mu \mathrm{l}$ each of the forward and reverse primers and $1 \mu \mathrm{l}$ of bisulfite-treated DNA was added to the mixture, for a total of $20 \mu \mathrm{l}$ of reaction system. The PCR reaction conditions were as follows: $95^{\circ} \mathrm{C}$ for $5 \mathrm{~min} ; 50$ cycles of $95^{\circ} \mathrm{C}$ for $1 \mathrm{~min}$, $60^{\circ} \mathrm{C}$ for $1 \mathrm{~min}$ and $72^{\circ} \mathrm{C}$ for $1 \mathrm{~min}$; followed by a cycle of $72^{\circ} \mathrm{C}$ for $1 \mathrm{~min}$ and termination at $4^{\circ} \mathrm{C}$. The PCR products were purified and $50 \mathrm{ng}$ of the resulting products was prepared for sequencing. The sequencing results were analyzed using ABI Sequence Scanner (http://www.appliedbiosystems.com/ absite/us/en/home.html). The methylation levels for each $\mathrm{CpG}$ site within the DNA amplicon were quantified by measuring the ratio between peak height values of guanine $(G)$ and adenine (A). In addition, to explore the effect of methylation 
A
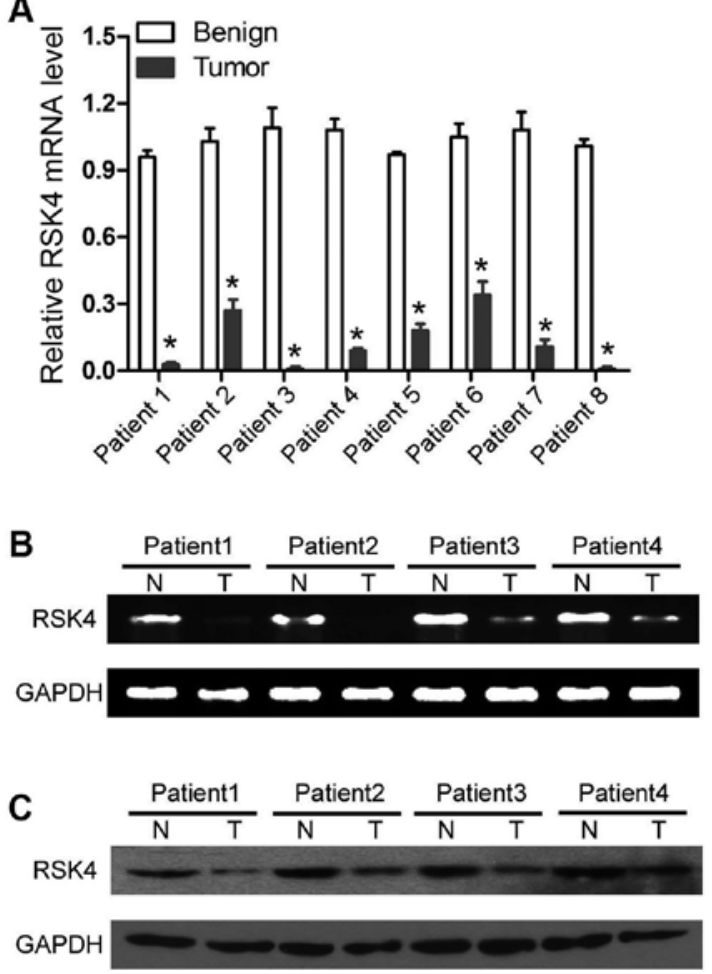

Figure 1. RSK4 expression in breast cancer tissues. (A) Real-time RT-PCR analysis of the mRNA expression of RSK4 in breast cancer tissues and adjacent benign tissues from 8 breast cancer patients. GAPDH was used as an internal control. The mRNA level of RSK4 in cancerous breast tissues was significantly decreased in all 8 patients compared with corresponding adjacent benign tissues ( $\left.{ }^{*} \mathrm{P}<0.05\right)$; (B) Gel-based RT-PCR analysis of the expression of RSK4 in breast cancer and adjacent benign tissues from 8 breast cancer patients, and GAPDH was used as an internal control. The RT-PCR product was separated on $2 \%$ agarose gels and the gel image was analyzed and quantified using ImageQuant software version 5.2 (Molecular Dynamics, Sunnyvale, CA, USA). (C) Western blot analysis of RSK4 protein level in breast cancer and adjacent benign tissues of the same patients, and GAPDH was used as an internal control. RSK4 protein levels in breast cancer tissues were also significantly lower than levels in the adjacent benign tissues.

on RSK4 expression and metastasis of human breast cancer, MDA-MB-231 cells were treated with the demethylation reagent 5-Aza-2'-deoxycytidine (5-Aza-CdR). The methylation sequencing was then performed and qPCR was used to detect the mRNA expression of RSK4 in the MDA-MB-231 cells.

Nude mouse tumorigenicity assay. For analyzing the RSK4 involvement in tumorigenicity in severe combined immunodeficient mice, the vector group and MDA-MB-231-RSK4 cells were collected, and washed 3 times with PBS. A total of 12 female athymic nude mice, 5-6 weeks old (18-22 g) were purchased from Beijing Slac Laboratory Animal Co. Ltd. (Beijing, China), and maintained in high efficiency particulate air-filtered cages in a pathogen-free facility. Mice were fed autoclaved diet with $14 \%$ protein and $3 \%$ fat. MDA-MB-231-RSK4 cells $\left(2 \times 10^{6}\right)$ were injected into the mammary fat pad of 6 female nude mice. The vector group cells were injected into the mammary fat pad of another 6 female nude mice. Mice infected with the breast cancer cells were kept in laminar flow animal rooms with stable humidity and temperature. Estradiol supplementation $(0.4 \mathrm{mg} / \mathrm{kg})$ was given every week after cell injection. After injection, tumor

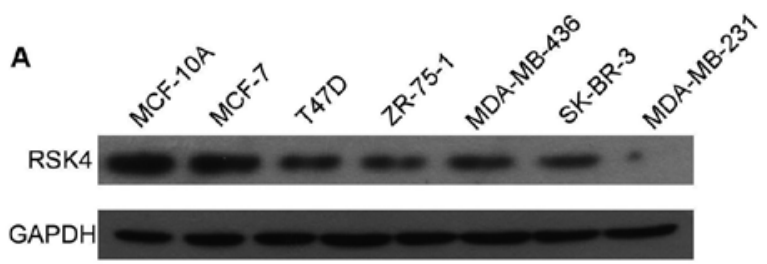

B

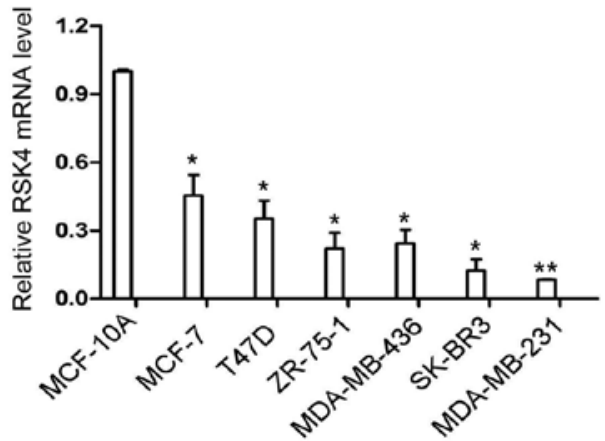

Figure 2. RSK4 expression in breast cancer cell lines. (A) Western blot analysis of RSK4 protein level in one normal human epithelial mammary cell line MCF-10A and 6 breast cancer cell lines MCF-7, T47D, ZR-75-1, MDA-MB-436, SK-BR-3 and MDA-MB-231. The protein level of RSK4 in all 6 breast cancer cell lines was lower than that in MCF-10A. (B) Real-time RT-PCR analysis of RSK4 mRNA levels in the cell lines. mRNA levels in these breast cancer cell lines were significantly decreased compared with the normal mammary cell line MCF-10A. ${ }^{*} \mathrm{P}<0.05,{ }^{* *} \mathrm{P}<0.01$.

appearance in mice was inspected each day by observation and palpation for 6 weeks, and tumor growth was measured weekly using a digital caliper (Shanghai Jiang Lai Biotechnology Co., Ltd., Shanghai, China). At the end of 6 weeks, all mice were sacrificed by cervical dislocation, and the necropsy was performed to check the presence of tumor nodules.

Statistical analysis. SPSS software, version 19.0 (IBM SPSS, Armonk, NY, USA) was used for the statistical analysis. The results are presented as mean \pm standard deviation values. A paired t-test was used to analyze the differences between tumor and adjacent non-tumor tissues. One way analysis of variance was used to analyze differences between more than three groups. $\mathrm{P}<0.05$ was considered to indicate a statistically significant difference.

\section{Results}

RSK4 expression in breast cancer tissues. To investigate the expression of RSK4 in tissues, breast cancer tissues and adjacent benign tissues from 8 breast cancer patients were collected and subjected to mRNA and protein level measurement. By real-time PCR and RT-PCR, we found that the mRNA level of the RSK4 gene in cancerous breast tissues was significantly decreased in all 8 patients compared with the corresponding adjacent benign tissues $(\mathrm{P}<0.05$; Fig. 1A and B). Western blot analysis was performed to determine the RSK4 protein levels between breast cancer tissues and adjacent benign tissues of the same patients, and GAPDH was used as an internal control. Consistent with the mRNA levels, the RSK4 protein levels in breast cancer tissues were also significantly lower than these levels in the 

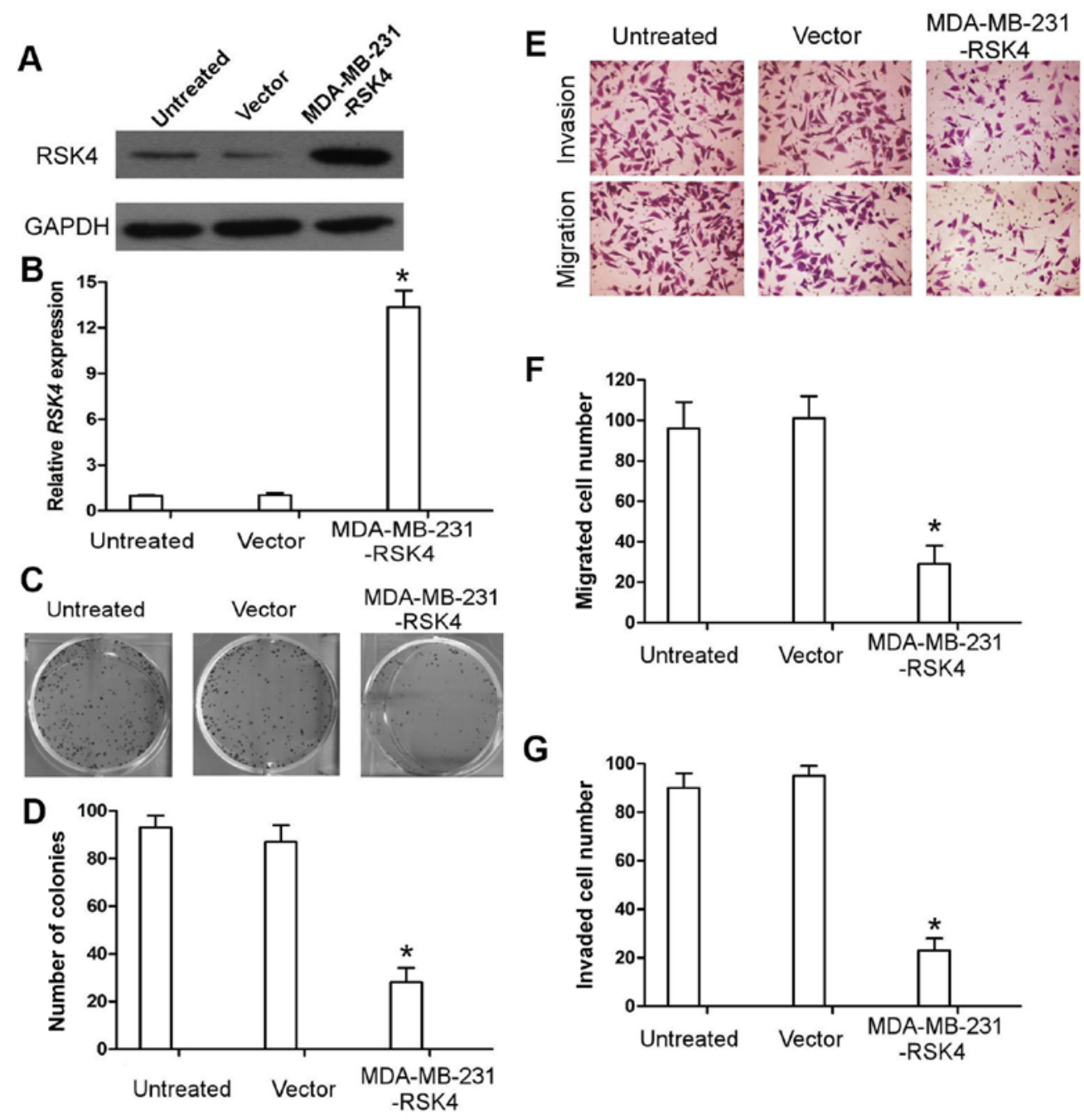

$\mathbf{F}$

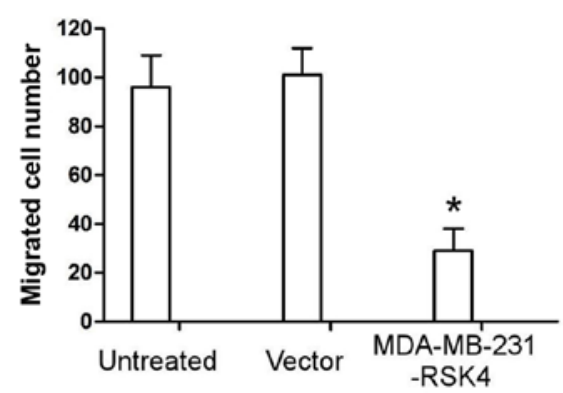

G

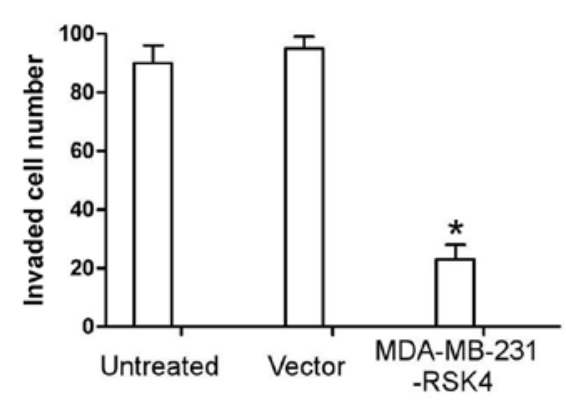

Figure 3. (A) Overexpression of RSK4 protein was confirmed by western blot analysis. (B) The overexpression of RSK4 mRNA was confirmed by real-time RT-PCR. (C and D) Colony formation assay indicated that the colony number in MDA-MB-231-RSK4 cells was significantly less than this number in the untreated or vector groups, "P<0.05. (E-G) Migration and invasion assays indicated that the migration and invasion potentials of MDA-MB-231-RSK4 cells were also markedly lower than that noted in the two control groups, ${ }^{*} \mathrm{P}<0.05$.

adjacent benign tissues (Fig. 1C). The expression level results clearly showed that the expression of RSK4 was markedly inhibited in the breast cancer tissues.

RSK4 expression in breast cancer cell lines. For further confirmation of RSK4 expression in breast cancer, we measured the mRNA and protein levels of RSK4 in 6 breast cancer cell lines, MCF-7, T47D, ZR-75-1, MDA-MB-436, SK-BR-3 and MDA-MB-231, and one normal human epithelial mammary cell line MCF-10A. As shown in Fig. 2A, the protein levels of RSK4 in all 6 breast cancer cell lines were lower than the level in the normal cell line. Among all 6 breast cancer cell lines, the highly invasive human breast carcinoma cell line MDA-MB-231 exhibited the most significant decrease in the RSK4 protein level compared with other 5 breast cancer cell lines, indicating that the expression level of RSK4 may possess a certain correlation with breast tumor progression (Fig. 2A). Consistence with the protein level, the result of qPCR also revealed a similar decrease in the RSK4 mRNA expression in these breast cancer cell lines, compared with the normal mammary cell line MCF-10A ( $\mathrm{P}<0.05, \mathrm{P}<0.01$; Fig. 2B).
Overexpression of $R S K 4$ in MDA-MB-231 cells. To explore the pathological role of RSK4 protein in breast cancer carcinogenesis and the underlying mechanisms, MDA-MB-231 cells with overexpression of RSK4 (MDA-MB-231-RSK4) were prepared using a lentivirus expression system. Untreated MDA-MB-231 cells (untreated) and MDA-MB-231 cells transfected with blank vectors (vector) were used as controls. The overexpression of RSK4 mRNA and protein was confirmed by western blot analysis (Fig. 3A) and real-time quantitative PCR (Fig. 3B), respectively. The MDA-MB-231-RSK4 cells showed high expression of RSK4 mRNA and protein in comparison with the untreated MDA-MB-231 cells or blank vector-transfected cells $(\mathrm{P}<0.05)$, showing that RSK4 protein was successfully overexpressed in the MDA-MB-231 cells. Then, we revealed that the proliferation of MDA-MB-231-RSK4 cells was significantly inhibited by overexpression of RSK4, as indicated by the reduced colony number in the MDA-MB-231-RSK4 cells by colony formation assay $(\mathrm{P}<0.05$; Fig. $3 \mathrm{C}$ and $\mathrm{D})$. Moreover, cell migration and invasion assays showed that the migration and invasion potentials of the MDA-MB-231-RSK4 


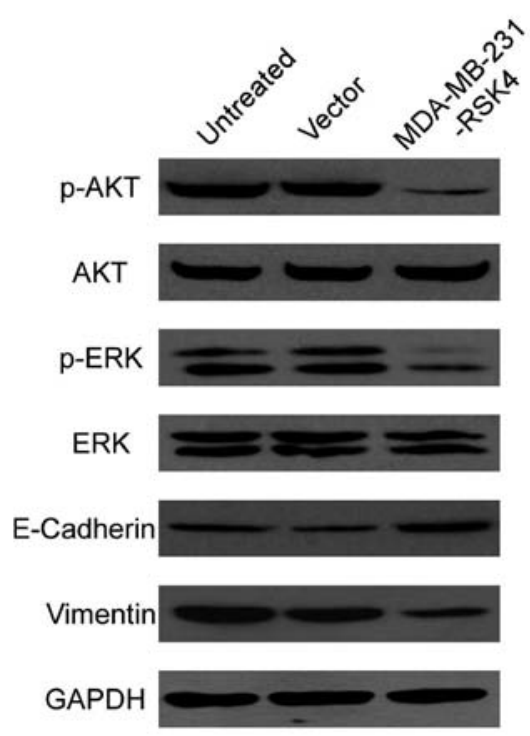

Figure 4. Western blot analysis of AKT and ERK signaling pathway proteins and two markers (E-cadherin and vimentin) for EMT in untreated cells, vector-transfected cells and MDA-MB-231-RSK4 cells. GAPDH was used as an internal control. The protein levels of AKT and ERK were not significantly altered by overexpression of RSK4 in the MDA-MB-231 cells, while the phosphorylated forms of AKT and ERK proteins were significantly decreased in the MDA-MB-231-RSK4 cells. E-cadherin was markedly increased in the RSK4-overexpressing MDA-MB-231 cells, while expression level of vimentin was much lower than the two control groups. EMT, epithelial mesenchymal transition; ERK, extracellular signal-regulated kinase.

cells were also markedly lower than the two control groups $(\mathrm{P}<0.05$; Fig. 3E-G).

AKT and ERK signaling pathways and EMT. As described before, previous studies have revealed the involvement of RSK proteins in AKT and ERK signaling pathway transduction, and RSK4 was also found to regulate ERK activation $(6,7,15)$. To further explore the molecular pathways mediating the regulation of breast cancer proliferation, migration and invasion by RSK4 protein, we analyzed the expression levels of several AKT and ERK signaling pathway proteins AKT and ERK, as well as the phosphorylation of AKT and ERK. Although the protein levels of AKT and ERK were not significantly changed by overexpressing RSK4 in the MDA-MB-231 cells, we found that the phosphorylated forms of AKT and ERK proteins were greatly decreased in the MDA-MB-231-RSK4 cells, clearly showing that RSK4 overexpression in this breast cancer cell line could inhibit the phosphorylation of major components of AKT and ERK signalling pathway cascades (Fig. 4). Moreover, E-cadherin and vimentin have been established as two important markers for EMT, which is critical for cell migration and cancer invasion. Here, we found that the expression level of E-cadherin was markedly increased in the RSK4-overexpressing MDA-MB-231 cells, while the expression level of vimentin was much lower than the two control groups, indicating that the role of RSK4 protein in regulating cancer metastasis may be mediated by EMT (Fig. 4).

Methylation of the RSK4 promoter in breast cancer cells. To further analyze the molecular mechanisms mediating RSK4 expression regulation in tumorigenesis, we analyzed the methylation of the RSK4 promoter in MDA-MB-231 cells (control cells) and 5-Aza-CdR-treated MDA-MB-231 cells (demethylated cells) by sequencing. As shown in Fig. 5A, methylation was represented by black circles and white circles represented an unmethylation status. The sequencing results showed that a high methylation level of RSK4 was found in the control cells, and the RSK4 methylation level in demethylated cells was significantly lower than that in the control cells. Consistently, the mRNA level of RSK4 in demethylated cells was markedly higher than that in the control cells $(\mathrm{P}<0.05$; Fig. 5B). In addition, the mRNA level of RSK4 in the demethylated cells was increased by 4 - to 5 -fold $(\mathrm{P}<0.05 ;$ Fig. 5B). Taking together, these results showed that the RSK4 gene expression in breast cancer cells was inhibited by DNA methylation in its promoter area.

RSK4 expression in mouse tumorigenicity. To further confirm the role of RSK4 in breast cancer development, the vector group and MDA-MB-231-RSK4 cells were applied for tumorigenicity assay in female athymic nude mice. The total body weight of the mice in each group is shown in Fig. 6B. Compared with the mice infected with the vector group, the tumors formed in the nude mice injected with MDA-MB-231-RSK4 cells were significantly reduced in weight and volume from week 3 to 6 (Fig. 6A, C and D), and showed a time-dependent reduction in tumor growth. This mouse model showed that overexpression of RSK4 in breast cancer cells could significantly suppress breast tumorigenicity.

\section{Discussion}

Breast cancer, usually characterized by a painful lump, change in breast shape, nipple fluid or red scaly patch of skin on the breast, is still the leading female cancer worldwide (1). Women with breast cancer may also suffer from bone pain, breath shortness, swollen lymph nodes and yellow skin caused by distant metastasis of the breast cancer (18). Although great progress has been achieved in reducing breast cancer incidence and death rate in developed countries, the need to intensively study its pathological mechanisms and develop more applicable and affordable early screening and therapy methods is crucial for developing countries with limited medical resources (18).

Novel cancer biomarkers with high efficiency and specificity have caused more and more attention in recent years, considering their potential application in the early screening and accurate diagnosis as well as personalized therapeutic strategy design (19). The rational application of molecular biomarkers may assure that breast cancer patients are given optimal treatment. Successfully, several molecular markers such as estrogen receptor, progesterone receptor, human epidermal growth factor receptor 2, antigen Ki-67 and $\% \mathrm{~S}$ phase cells, have been applied for classification of heterogeneous breast cancers (19). Recent publications have also revealed the potential of several other molecules such as cyclin E, B-Myb, Twist, and DMP1 $\beta$ as novel therapeutic targets for breast cancer patients (19). In the present study, we revealed that RSK4 expression was greatly repressed in breast cancer cells and tissues, and overexpression of RSK4 in breast cancer cells inhibited cell migration. Moreover, a mouse model experiment by overexpressing of RSK4 in breast cancer 
A

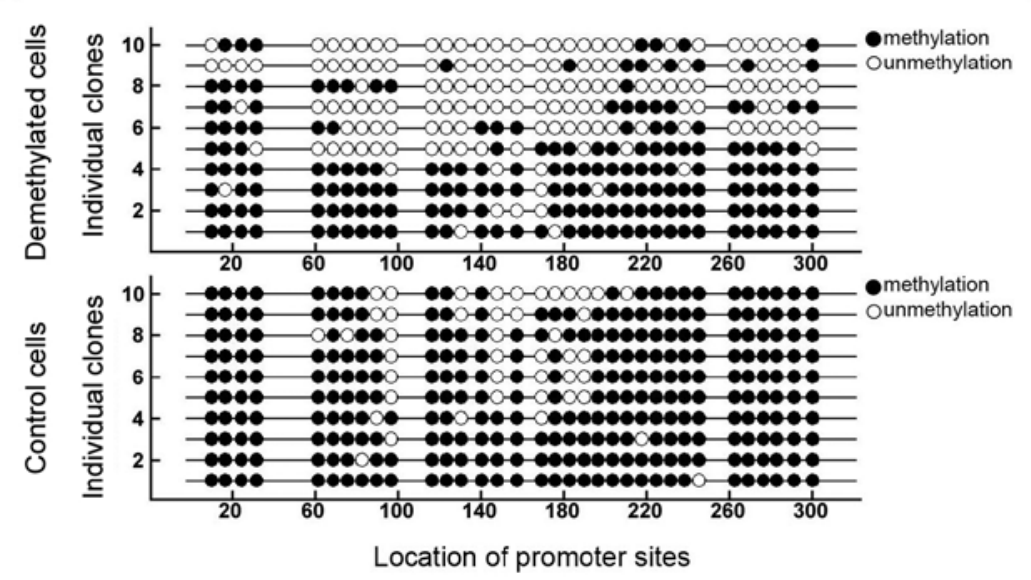

B

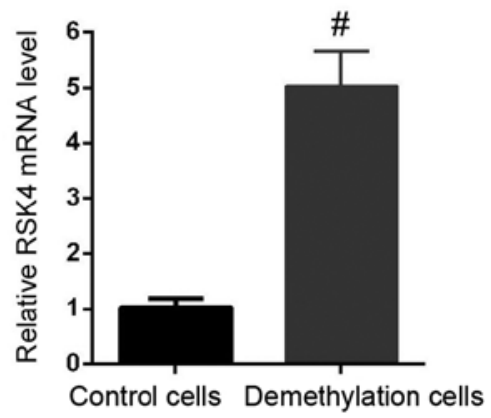

Figure 5. (A) DNA methylation sequencing. Methylation of the RSK4 promoter in breast cancer cells (control cells) and 5-Aza-CdR-treated MDA-MB-231 cells (demethylated cells) by bisulfite direct sequencing assay. Methylation is represented by black circles and white circles represent unmethylation. (B) Real-time RT-PCR analysis of RSK4 mRNA expression in control and demethylated MDA-MB-231 cells. The RSK4 mRNA in demethylation cells was significantly higher than control cells, ${ }^{*} \mathrm{P}<0.05$.

A

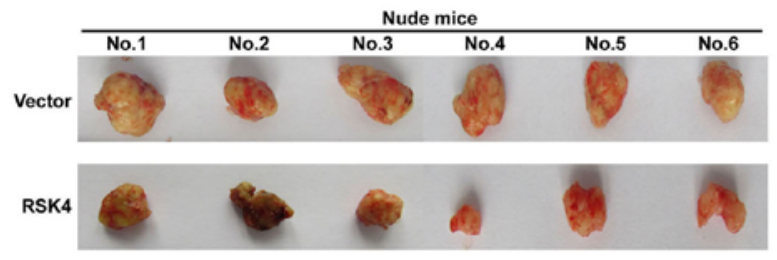

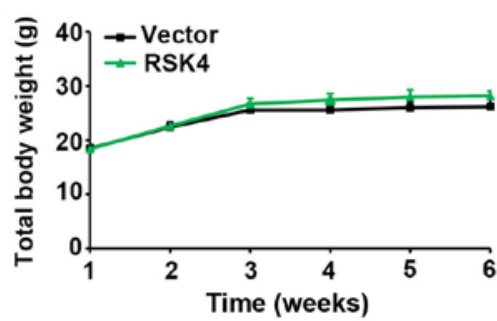

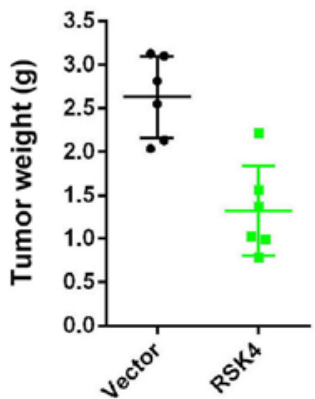

D

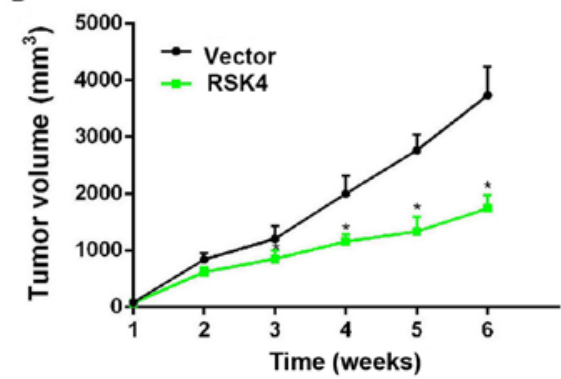

Figure 6. (A) Nude mouse tumorigenicity assay. The nude mice were infected with the vector group and MDA-MB-231-RSK4 cells, respectively. (B) Total body weight $(\mathrm{g})$ in two groups. (C) Tumor weight $(\mathrm{g})$ in two groups. The tumors formed in nude mice injected with MDA-MB-231-RSK4 cells were significantly reduced in weight compared with the nude mice injected with vector. (D) Tumor volume $\left(\mathrm{mm}^{3}\right)$ in the two groups. The tumors formed in nude mice injected with MDA-MB-231-RSK4 cells were significantly reduced in volume compared with the nude mice injected with the vector, ${ }^{*} \mathrm{P}<0.05$.

cells further confirmed its critical role in regulating breast cancer progression. Combined with other publications about RSK4 and breast cancer $(3,13,14)$, recent evidence provides an important research basis for the future application of RSK4 in breast cancer screening, diagnosis and therapy, although large prospective clinical studies should be performed to establish its predictive value in patients with breast cancer.

Comprehensive understanding of breast cancer progression and breast cell transformation may provide critical insight for the development of reliable diagnostic and therapeutic methods for breast cancer treatment. AKT and ERK signaling pathways have long been established as central mediators in the development of multiple cancers by regulating cancer cell growth, survival, proliferation and migration (20,21). Raf/MEK/ERK and PI3K/PDK1/Akt signaling pathways have been associated with breast cancer development and it resistance to cytotoxic drugs (22). In view of the significant role of the AKT and ERK signaling pathways in breast cancer, they have been extensively applied for development of targeted therapy for breast cancer patients, and multiple promising therapeutic agents targeting the AKT and ERK signaling pathways are under evaluation in clinical trials (23). In the present study, we found that the involvement of RSK4 in breast cancer progression is mediated by the AKT and ERK signaling pathways, which provide meaningful insight into the pathological mechanisms of breast cancer and provide more choices for the development of new 
therapies. In addition, EMT is a cellular program that is closely associated with lung tumorigenesis and the majority of female cancer deaths are caused by cell invasion (24). In the present study, we found that the expression levels of E-cadherin and vimentin were markedly altered in RSK4-overexpressing MDA-MB-231 cells, showing that RSK4 protein may regulate EMT in breast cancer and may be used as biomarkers for identification of breast tumor subsets with greater risk occurrence.

Recent research has also pinpointed the increasing roles of epigenetic processes such as DNA methylation in breast cancer pathogenicity, prevention and treatment (25). For instance, the methylation of adenomatous polyposis coli (APC), APC/C activator $\mathrm{CDH} 1$ and catenin $\beta-1$ (CTNNB1) genes has been associated with breast cancer development (26). Also, the hypomethylation of Alu and LINE-1 promoter regions has been shown to be linked with the HER-enriched breast cancer subtype (27). Furthermore, the BRCA1 gene is also subjected to hypermethylation which regulates the gene expression pattern during breast cancer development (28). Here, we found that the inhibition of RSK4 expression in breast cancer progression was mediated by the methylation in its promoter regions, attaching more importance to the epigenetic mechanisms in breast cancer pathology which may lead to improved diagnosis and treatment of this disease.

In conclusion, we revealed that RSK4 expression was markedly suppressed in breast cancer cells and tissues, and overexpression of RSK4 in breast cancer cells inhibited cell migration and invasion. A mouse model experiment of overexpression of RSK4 further confirmed its critical role in regulating breast cancer progression. The involvement of RSK4 in breast cancer was mediated by the AKT and ERK signaling pathways and the expression of RSK4 was altered by DNA methylation in promoter regions. These results provide important insight into the role of RSK4 in breast cancer development and may help improve breast cancer prevention, diagnosis and treatment.

\section{Acknowledgements}

The present study was funded by the Guangxi Scientific and Technology Research Project Foundation (14124004-1-12) and the National Natural Science Foundation of China (NSFC81260394).

\section{References}

1. Torre LA, Bray F, Siegel RL, Ferlay J, Lortet-Tieulent J and Jemal A: Global cancer statistics, 2012. CA Cancer J Clin 65: 87-108, 2015

2. Zeng H, Zheng $R$, Zhang S, Zou $X$ and Chen W: Incidence and mortality of female breast cancer in China, 2009. Thorac Cancer 4: 400-404, 2013.

3. Thakur A, Rahman KW, Wu J, Bollig A, Biliran H, Lin X, Nassar H, Grignon DJ, Sarkar FH and Liao JD: Aberrant expression of X-linked genes RbAp46, Rsk4, and Cldn2 in breast cancer. Mol Cancer Res 5: 171-181, 2007.

4. Yntema HG, van den Helm B, Kissing J, van Duijnhoven G, Poppelaars F, Chelly J, Moraine C, Fryns JP, Hamel BC, Heilbronner $\mathrm{H}$, et al: A novel ribosomal S6-kinase (RSK4; RPS6KA6) is commonly deleted in patients with complex X-linked mental retardation. Genomics 62: 332-343, 1999.

5. Kohn M, Hameister H, Vogel $M$ and Kehrer-Sawatzki $H$ : Expression pattern of the Rsk2, Rsk4 and Pdk1 genes during murine embryogenesis. Gene Expr Patterns 3: 173-177, 2003.
6. Myers AP, Corson LB, Rossant J and Baker JC: Characterization of mouse Rsk4 as an inhibitor of fibroblast growth factor-RAS-extracellular signal-regulated kinase signaling. Mol Cell Biol 24: 4255-4266, 2004.

7. Dümmler BA, Hauge C, Silber J, Yntema HG, Kruse LS Kofoed B, Hemmings BA, Alessi DR and Frödin M: Functional characterization of human RSK4, a new 90-kDa ribosomal S6 kinase, reveals constitutive activation in most cell types. J Biol Chem 280: 13304-13314, 2005.

8. Niehof M and Borlak J: RSK4 and PAK5 are novel candidate genes in diabetic rat kidney and brain. Mol Pharmacol 67: 604-611, 2005.

9. López-Vicente L, Armengol G, Pons B, Coch L, Argelaguet E, Lleonart M, Hernández-Losa J, de Torres I, Ramon y Cajal S: Regulation of replicative and stress-induced senescence by RSK4, which is down-regulated in human tumors. Clin Cancer Res 15: 4546-4553, 2009.

10. Dewdney SB, Rimel BJ, Thaker PH, Thompson DM Jr, Schmidt A, Huettner P, Mutch DG, Gao F and Goodfellow PJ: Aberrant methylation of the X-linked ribosomal S6 kinase RPS6KA6 (RSK4) in endometrial cancers. Clin Cancer Res 17: 2120-2129, 2011.

11. Arechavaleta-Velasco F,Zeferino-Toquero M,Estrada-Moscoso I, Imani-Razavi FS, Olivares A, Perez-Juarez CE and Diaz-Cueto L: Ribosomal S6 kinase 4 (RSK4) expression in ovarian tumors and its regulation by antineoplastic drugs in ovarian cancer cell lines. Med Oncol 33: 11, 2016

12. López-Vicente L, Pons B, Coch L, Teixidó C, Hernández-Losa J, Armengol G and Ramon Y Cajal S: RSK4 inhibition results in bypass of stress-induced and oncogene-induced senescence. Carcinogenesis 32: 470-476, 2011.

13. Li Q, Jiang Y, Wei W, Ji Y, Gao H and Liu J: Frequent epigenetic inactivation of RSK4 by promoter methylation in cancerous and non-cancerous tissues of breast cancer. Med Oncol 31: 793, 2014.

14. Zhu J, Li QY, Liu JL, Wei W, Yang HW and Tang W: RSK4 knockdown promotes proliferation, migration and metastasis of human breast adenocarcinoma cells. Oncol Rep 34: 3156-3162, 2015.

15. Frödin $\mathrm{M}$ and Gammeltoft S: Role and regulation of $90 \mathrm{kDa}$ ribosomal S6 kinase (RSK) in signal transduction. Mol Cell Endocrinol 151: 65-77, 1999.

16. Roy $\mathrm{P}$ and Jacobson K: Overexpression of profilin reduces the migration of invasive breast cancer cells. Cell Motil Cytoskeleton 57: 84-95, 2004.

17. Cheng GZ, Chan J, Wang Q, Zhang W, Sun CD and Wang LH: Twist transcriptionally up-regulates AKT2 in breast cancer cells leading to increased migration, invasion, and resistance to paclitaxel. Cancer Res 67: 1979-1987, 2007.

18. DeSantis CE, Fedewa SA, Goding Sauer A, Kramer JL, Smith RA and Jemal A: Breast cancer statistics, 2015: Convergence of incidence rates between black and white women. CA Cancer J Clin 66: 31-42, 2016.

19. Inoue K and Fry EA: Novel molecular markers for breast cancer. Biomark Cancer 8: 25-42, 2016.

20. Sever R and Brugge JS: Signal transduction in cancer. Cold Spring Harb Perspect Med: Apr 1, 2015 (Epub ahead of print). doi: $10.1101 /$ cshperspect.a006098.

21. Yu Z, Ye S, Hu G, Lv M, Tu Z, Zhou K and Li Q: The RAF-MEK-ERK pathway: Targeting ERK to overcome obstacles to effective cancer therapy. Future Med Chem 7: 269-289, 2015.

22. Navolanic PM, Steelman LS and McCubrey JA: EGFR family signaling and its association with breast cancer development and resistance to chemotherapy (Review). Int J Oncol 22: 237-252, 2003.

23. Alvarez RH, Valero V and Hortobagyi GN: Emerging targeted therapies for breast cancer. J Clin Oncol 28: 3366-3379, 2010.

24. Felipe Lima J, Nofech-Mozes S, Bayani J and Bartlett JM: EMT in breast carcinoma - A review. J Clin Med 5: 65, 2016.

25. Basse $\mathrm{C}$ and Arock $\mathrm{M}$ : The increasing roles of epigenetics in breast cancer: Implications for pathogenicity, biomarkers, prevention and treatment. Int J Cancer 137: 2785-2794, 2015.

26. Hoque MO, Prencipe M, Poeta ML, Barbano R, Valori VM, Copetti M, Gallo AP, Brait M, Maiello E, Apicella A, et al: Changes in $\mathrm{CpG}$ islands promoter methylation patterns during ductal breast carcinoma progression. Cancer Epidemiol Biomarkers Prev 18: 2694-2700, 2009.

27. Park SY, Seo AN, Jung HY, Gwak JM, Jung N, Cho NY and Kang GH: Alu and LINE-1 hypomethylation is associated with HER2 enriched subtype of breast cancer. PLoS One 9: e100429, 2014.

28. Hedenfalk I, Duggan D, Chen Y, Radmacher M, Bittner M, Simon R, Meltzer P, Gusterson B, Esteller M, Kallioniemi OP, et al: Gene-expression profiles in hereditary breast cancer. N Engl J Med 344: 539-548, 2001. 